\title{
How should complete lung collapse secondary to primary spontaneous pneumothorax be managed? 䇺
}

\author{
Authors: Muhammad Badar Ganaie, ${ }^{\mathrm{A}}$ Usman Maqsood, ${ }^{\mathrm{B}}$ Simon Lea, ${ }^{\mathrm{C}}$ Michael John Bankart, ${ }^{\mathrm{D}}$ Shiva Bikmalla, \\ Muhammad Amin Afridi, ${ }^{E}$ Masood Ahmad Khalil, ${ }^{F}$ Imran Hussain ${ }^{A}$ and Mohammed Haris ${ }^{A}$
}

Management of primary spontaneous pneumothorax (PSP) depends on the symptoms and size of lung collapse. The British Thoracic Society recommends needle aspiration (NA) for all PSP requiring intervention, followed by intercostal drain (ICD) if NA fails. We compared the role of NA versus ICD as the first step in PSP with 'complete lung collapse'.

This was a retrospective observational study of 877 consecutive pneumothorax episodes at University Hospitals of North Midlands, Stoke on Trent, UK. Chest X-ray (CXR) at presentation was reviewed to identify PSP with complete lung collapse. The primary outcome measure was successful lung re-inflation after initial intervention.

Two-hundred and sixty-six PSP patients were identified; 69 had complete lung collapse on CXR of which 35 had NA and 34 had ICD. The ICD group had a significantly better immediate success compared with the NA group (62\% versus $11 \%$, odds ratio $(O R)=12.5, p<0.0001$; after adjustment for potential confounders, $O R$ increased to $26.4, p=0.0001$ ) although long-term outcomes were comparable.

There should be clear consensus on definition and management of complete lung collapse. PSP with complete lung collapse could be managed as a separate subgroup where ICD placement is considered to be the first intervention.

KEYWORDS: Pleural disease, primary spontaneous pneumothorax

\section{Introduction}

Primary spontaneous pneumothorax (PSP) is the presence of air in the pleural space with no history of trauma. PSP occurs in otherwise healthy individuals with no apparent lung disease,

Authors: ${ }^{A}$ Consultant respiratory physician, Royal Stoke University Hospital, Stoke-on-Trent, UK; ${ }^{B}$ consultant respiratory physician, Sandwell and West Birmingham Hospitals, West Bromwich, UK; ${ }^{C}$ Department of Research and Development, Royal Stoke University

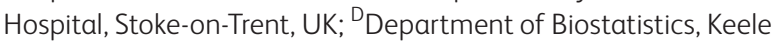
University, Stoke-on-Trent, UK; ${ }^{E}$ consultant respiratory physician, New Cross Hospital, Wolverhampton, UK; F consultant respiratory Physician, County Hospital, Stafford, UK although most individuals have underlying apical blebs or bullae predisposing them to pneumothorax. The annual incidence of PSP has been reported as $7.4-18$ per 100,000 for men and 1.2-6 per 100,000 for women. ${ }^{2,3}$ In 2015, there were 1,804 hospital admissions in UK for PSP in men and 564 in women, giving an admission-based rate of 8.2 per 100,000 for men and 2.5 per 100,000 for women, as highlighted by a recent large-scale UKbased epidemiological study. ${ }^{4}$ The annual mortality rate of PSP has been quoted as 1.26 per million for men and 0.62 per million for women. ${ }^{5}$ Recurrence rates of PSP are estimated to be up to $32 \%$, with the greatest risk in the in the first year. ${ }^{6}$

The first British Thoracic Society (BTS) guidelines for the management of spontaneous pneumothorax published in 1993 subdivided PSP into small, moderate and complete according to the size of pneumothorax on chest X-ray (CXR). Needle aspiration (NA) was advised as the first step in the management of symptomatic pneumothorax and/or those with complete lung collapse. ${ }^{7}$ Revised guidelines in 2003 once again recommended NA as the first step followed by a second NA attempt prior to intercostal drain (ICD) for all symptomatic and/or large PSP (size $>2 \mathrm{~cm}$ on CXR). ${ }^{8}$ Current 2010 BTS guidelines suggest NA followed by ICD if NA fails to re-inflate the lung. ${ }^{9}$ However, there is variation in practice with a BTS audit in 2010 showing that $42 \%$ of all PSP episodes requiring intervention had ICD as the first step without an initial attempt to perform NA. ${ }^{10}$

Most patients with PSP and complete lung collapse present with acute symptoms requiring intervention. NA alone may not be able to facilitate removal of air from the pleural cavity in those with complete lung collapse due to the presence of persistent air leak exceeding the catheter's ability to remove air. ${ }^{11}$ To the best of our knowledge, there are no studies looking at outcomes in PSP with complete lung collapse. The aim of this study was to compare the outcome of first intervention (NA versus ICD) in patients with PSP and complete lung collapse.

\section{Methods}

This was a retrospective observational study of 877 consecutive pneumothorax episodes presenting to the University Hospitals of North Midlands, Stoke-on-Trent, UK between March 2008 and February 2015. After excluding secondary spontaneous pneumothorax (SSP), traumatic, postoperative and iatrogenic pneumothorax, 266 PSP episodes remained. CXR images on picture archiving and communication system (PACS) were 
reviewed by two independent respiratory physicians. There was excellent correlation between the assessors and they were blinded to the eventual patient outcomes. Outcome measures, obtained from patient care record, included successful lung re-inflation after first intervention, length of hospital stay, further interventions including surgery (if performed) and recurrence rate within 1 year.

\section{Data collection}

Patients were identified by the International Classification of Diseases, $10^{\text {th }}$ revision (ICD-10) using the diagnosis codes; 393.0 (spontaneous tension pneumothorax), 393.1 (other spontaneous pneumothorax), 393.8 (other pneumothorax) and 393.9

(pneumothorax, unspecified). Electronic patient records and case notes from the emergency department and inpatient episodes were reviewed.

\section{Definitions}

PSP was defined according to the current BTS guidelines; spontaneous pneumothorax in those aged less than 50 years and no significant smoking history ( $<20$ pack years) or evidence of underlying lung disease clinically or radiologically. ${ }^{9}$

Complete lung collapse was defined in line with the Spanish Society of Pulmonology and Thoracic Surgery (SEPAR) guidelines as complete separation of the visceral and parietal pleura from the base to the apex of the pleural space associated with uniform formation of a stump (Fig 1). ${ }^{12}$

Successful lung re-inflation was defined as sustained complete or near complete lung re-expansion on CXR, and not requiring further intervention.

\section{Statistical Analysis}

Categorical variables ( $\mathrm{n}, \%$ ) were analysed using Fisher's exact test. Continuous data (median, $25^{\text {th }}-75^{\text {th }}$ percentile) were compared using the Wilcoxon rank sum test. P-values $<0.05$ were considered statistically significant. A multivariable logistic regression was used to assess the hypothesis of no difference in proportion of successes (in complete lung re-inflation) comparing NA and ICD as the first method, with adjustment for potential confounders (mentioned below).

\section{Results}

A total of 877 patients with pneumothorax were identified between March 2008 and February 2015, of which 266 (30\%) were PSP, 229 (26\%) were SSP, 341 (39\%) were traumatic or postoperative and 41 (5\%) were iatrogenic pneumothorax. SSP, traumatic, postoperative, iatrogenic pneumothorax and PSP
Fig 1. Right sided primary spontaneous pneumothorax with complete lung collapse

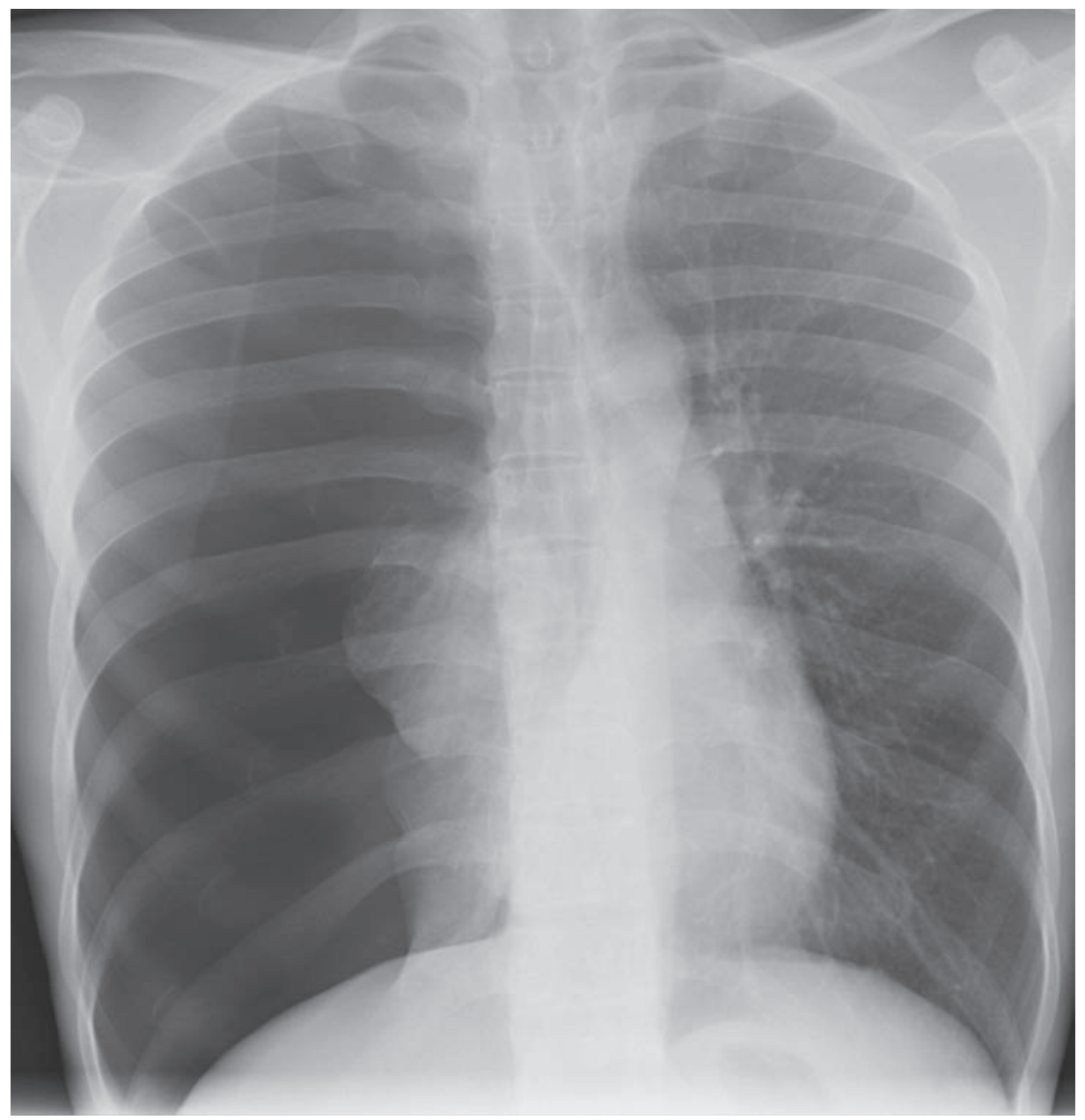


patients referred for cardiothoracic surgery from other centres were excluded from further analysis.

Of the 266 patients with PSP, 69 were identified as having complete lung collapse on CXR, of which 35 patients had NA and 34 had ICD as the first intervention. Four patients in the NA group and six in the ICD group had one previous episode of pneumothorax; no data was available regarding previous treatment modalities. There were no statistically significant differences between the two groups in terms of age, sex, smoking history and symptoms at the time of presentation (Table 1).

Compared with the NA group, patients in the ICD group had a significantly better success rate (62\% (ICD) versus $11 \%$ (NA), $\mathrm{OR}=12.5, \mathrm{p}<0.0001)$. Thirty-one patients that failed NA required ICD and were admitted. Two patients who failed NA had further unsuccessful NA attempts (one patient had one further attempt and the second patient had two further attempts). Of the 31 NA failures, all required ICD (five patients had two ICDs and one patient had three ICDs) with 10 patients needing surgical intervention. In the ICD group, 12 patients had multiple ICDs (nine patients had two ICDs and two patients had three ICDs) of which eight patients had persistent air leak that required surgical intervention.

In the ICD and NA groups, median length of stay (LOS) (7 days (ICD) versus 6 days (NA), $p=0.18$ ) and recurrence within 1 year ( $3 \%$ (ICD) versus $11 \%(N A), p=0.36$ ) were not significantly different. Median LOS was significantly better if NA was successful $(n=4)$ compared with successful ICD $(n=21)$ (1 day (NA) versus 6 days (ICD), $p=0.0018$ ). Around a third of patients in both groups
(38\% (ICD) versus $29 \%$ (NA), $p=0.45$ ) required a definitive surgical intervention during the same admission.

A multivariable logistic regression compared the proportion of successes in the two groups. The following confounders were included in the model based on clinical relevance and availability: age, sex, smoking, cough and chest pain. A single model was run, and all predictors were retained in the model whether significant or not. The model produced an adjusted OR of $26.4(4.8,142.3)$, $p=0.0001$ for first method used, with a significantly greater proportion of successes in the ICD first group.

\section{Discussion}

Despite PSP being a common presentation, there is lack of consensus between various international societal guidelines on the initial management based on the symptoms and degree of collapse on CXR (Table 2).

Our data suggest that NA is less successful than ICD in PSP with complete lung collapse. Performing NA in this subgroup of patients, which is more likely to fail, results in additional invasive procedures leading to heightened patient anxiety, further radiation exposure due to repeated imaging and extra nursing / medical input. NA should still be considered first line in PSP patients who do not have complete lung collapse (Fig 2) as it is less invasive, simple to perform and has a high success rate leading to reduced hospital stay.

The role of NA compared with ICD as a first step in the management of PSP with complete lung collapse as a subgroup

Table 1. Comparison of intercostal drain and needle aspiration as first intervention in the management of

primary spontaneous pneumothorax with complete lung collapse

\begin{tabular}{|c|c|c|c|}
\hline & ICD as first intervention $(n=34)$ & NA as first intervention $(n=35)$ & $\mathrm{p}$-value \\
\hline Median age, years (IQR) & $30.5(26-35)$ & $30(24-38)$ & 0.49 \\
\hline Male, n (\%) & $26(76 \%)$ & $24(69 \%)$ & 0.59 \\
\hline Right sided, n (\%) & $20(59 \%)$ & $21(60 \%)$ & $>0.99$ \\
\hline Previous pneumothorax, n (\%) & $6(18 \%)$ & $4(11 \%)$ & 0.51 \\
\hline \multicolumn{4}{|l|}{ Smoking history } \\
\hline Current/ex, n (\%) & $25(74 \%)$ & $29(82 \%)$ & 0.39 \\
\hline Never, n (\%) & $6(18 \%)$ & $3(9 \%)$ & 0.31 \\
\hline Not available, n (\%) & $3(8 \%)$ & $3(9 \%)$ & $>0.99$ \\
\hline Mean pack years, $n$ (IQR) & $12.5(8.5-20)$ & $9(5-20)$ & 0.49 \\
\hline \multicolumn{4}{|l|}{ Symptoms } \\
\hline Chest pain, $\mathrm{n}(\%)$ & $29(85 \%)$ & $29(83 \%)$ & $>0.99$ \\
\hline Dyspnoea, n (\%) & $33(97 \%)$ & $30(86 \%)$ & 0.20 \\
\hline Cough, n (\%) & $11(32 \%)$ & $7(20 \%)$ & 0.28 \\
\hline Successful lung re-inflation, n (\%) & $21(62 \%)$ & $4(11 \%)$ & $<0.0001$ \\
\hline Median LOS, days (IQR) & $7(4-13)$ & $6(4-10)$ & 0.18 \\
\hline LOS if surgical intervention, days (IQR) & $13(7.5-16.5)$ & $10(8-13)$ & 0.55 \\
\hline LOS if no surgical intervention, days (IQR) & $6(3-9.5)$ & $5(3-6.5)$ & 0.32 \\
\hline Surgical intervention, $\mathrm{n}(\%)$ & $13(38 \%)$ & $10(29 \%)$ & 0.45 \\
\hline Recurrence at 1 year, n (\%) & $1(3 \%)$ & $4(11 \%)$ & 0.36 \\
\hline
\end{tabular}

$I C D=$ intercostal drain; $I Q R=$ interquartile range; $L O S=$ length of stay; $N A=$ needle aspiration; $P S P$ = primary spontaneous pneumothorax. 
Table 2. Summary of recommendations for the initial management of primary spontaneous pneumothorax by different societal guidelines

\section{International guidelines}

American College of Chest Physicians consensus statement, 2001. ${ }^{13}$

Belgian Society of Pneumology guidelines, $2005 .^{14}$

Spanish Society of Pulmonology and Thoracic Surgery guidelines, 2008. ${ }^{12}$

Japan Society for Pneumothorax and Cystic Lung Diseases guidelines, 2009. ${ }^{15}$

British Thoracic Society Pleural Diseases guidelines, 2010.9

European Respiratory Society Task Force Not available. consensus statement, 2015. ${ }^{16}$

\section{Classification of PSP}

Large $>3 \mathrm{~cm}$ apex-to-cupola distance, on CXR.

Large with lung dehiscence over the whole length of lateral chest wall on CXR.

Partial, complete and complete with total lung collapse on CXR.

Mild, moderate and severe according to the degree of collapsed lung on CXR.

Large $>2 \mathrm{~cm}$ at the level of hilum, on CXR.

\section{Treatment recommendations}

ICD for clinically unstable or large PSP.

Observation for small and minimally symptomatic PSP, NA or small-bore ICD for symptomatic and/or large PSP.

NA or small-bore ICD for patients with breathlessness or complete PSP.

Observation or NA for mild PSP, ICD for moderate to severe PSP.

NA as first intervention for large PSP and/ or breathless patients.

NA or small-bore ICD attached to one-way valve, as first intervention.

CXR = chest X-ray; ICD = intercostal drain; NA = needle aspiration; PSP = primary spontaneous pneumothorax.

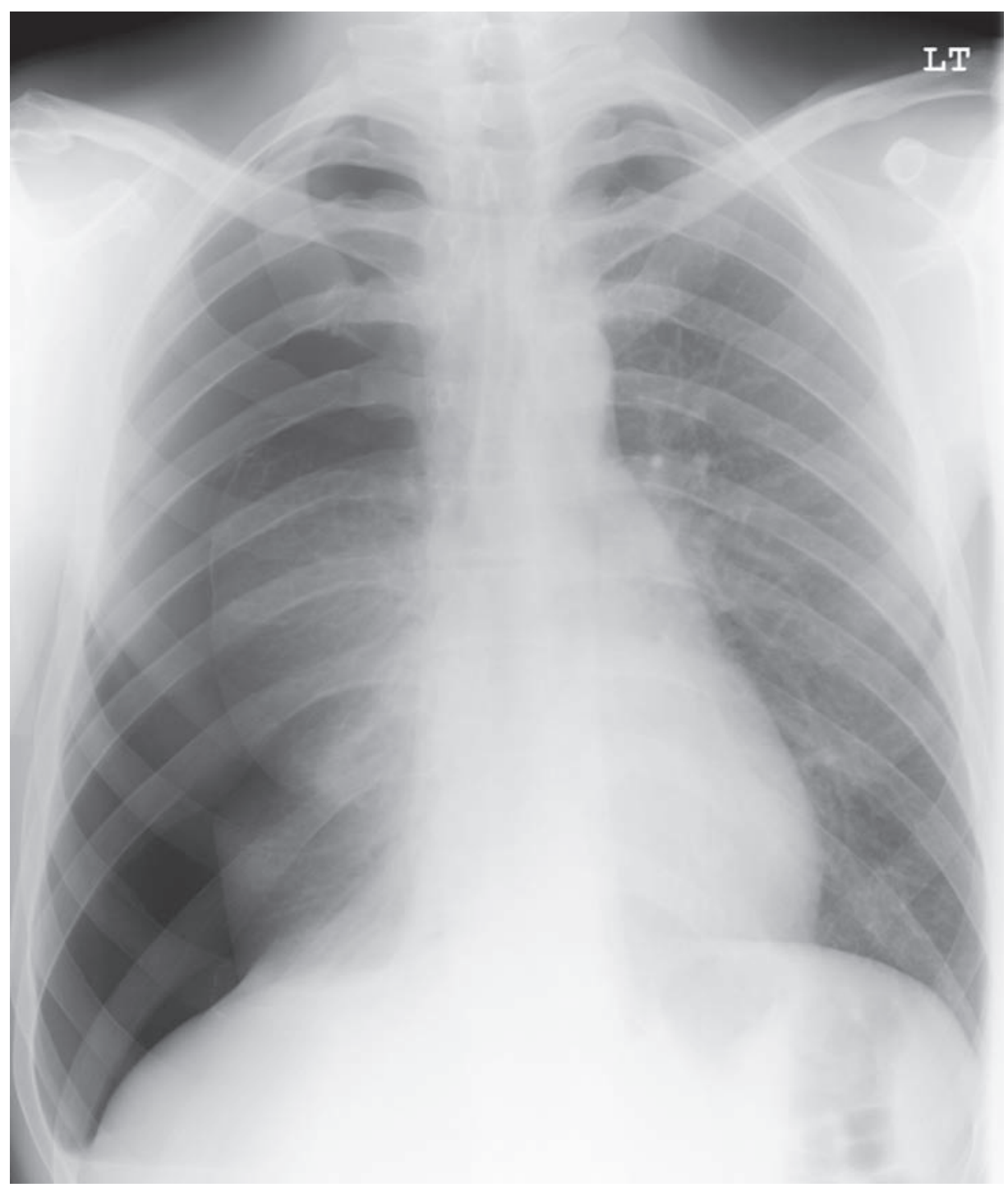


Table 3. Success rates of needle aspiration (and intercostal drain if available) reported in previous primary spontaneous pneumothorax studies

\begin{tabular}{|c|c|c|c|c|}
\hline Study (year, country) & Study type & $\begin{array}{l}\text { Number of } \\
\text { patients }\end{array}$ & $\begin{array}{l}\text { NA immediate success } \\
\text { rate (ICD if available) }\end{array}$ & Predictors of failure of NA \\
\hline Raja et al ${ }^{17}$ (1981, UK) & Prospective & 33 & $69 \%$ & $N / A$ \\
\hline Harvey et al ${ }^{18}$ (1994, UK) & Prospective, multicentre & 73 & $80 \%$ & Size not predictor of failure \\
\hline Noppen et al ${ }^{19}$ (2002, Belgium) & Prospective, multicentre & 60 & $59 \%$ NA, $63 \%$ ICD & Repeat NA \\
\hline Packham et al ${ }^{20}$ (2003, UK) & Retrospective & 85 & $50 \%$ & Managed by general physicians \\
\hline Chan et al ${ }^{21}$ (2005, Hong Kong) & Retrospective & 91 & $50.5 \%$ & Size $>40 \%$ \\
\hline Camuset et al ${ }^{22}$ (2006, France) & Prospective & 35 & $69 \%$ & Size not predictor of failure \\
\hline Ayed et $a^{23}$ (2006, Kuwait) & Prospective & 137 & $62 \% \mathrm{NA}, 68 \%$ ICD & $N / A$ \\
\hline Nishiuma et $a^{24}$ (2012, Japan) & Retrospective & 143 & $72.5 \%$ & Size $>50 \%$ \\
\hline Thelle et $a l^{25}$ (2017, Norway) & Prospective, multicentre & 79 & $73 \%$ NA, $37 \%$ ICD & N/A \\
\hline
\end{tabular}

has not been studied before. Previous studies have compared the success of NA with ICD for all PSP requiring intervention (not just PSP with complete lung collapse as in our study); these studies reported no significant difference in the immediate success rate (Table 3). The failure rate of NA is reported to be between 20 $50 \%$ in these studies. Chan et al and Nishiuma et al reported an initial size of PSP of $>40-50 \%$ being a predictor of failed NA. ${ }^{21,24}$ Nikolić et al showed that all PSP patients with size $>5.4 \mathrm{~cm}$ at the level of the hilum initially managed with NA subsequently required ICD insertion. ${ }^{26}$ It is difficult to draw an overall conclusion from these studies because of limited patient numbers, varying study designs and treatment strategies (some studies included a second NA attempt after the first failed attempt).

A systematic review comparing NA with ICD in the initial management of PSP ( $n=194)$ concluded that NA was advantageous in the initial management of PSP because of shorter hospitalisation but there was no significant difference in recurrence at 1 year. ${ }^{27}$ Zehtabchi et al conducted an emergency medicine review comparing the efficacy and safety of NA with ICD in the management of PSP $(n=270)$. There was no significant difference between NA and ICD in outcomes of immediate failure, 1-week failure, risk of complications and 1-year recurrence rate, but NA was associated with lower rates of hospitalisation and reduced length of hospital stay. ${ }^{28}$ Systematic review and meta-analysis by Aguinagalde et al looking at the effectiveness of NA compared with ICD for treating PSP ( $n=331 ; 160$ had NA and 171 had ICD) reported ICD to be more effective in terms of immediate success, but this effect disappeared after 1 week and did not persist in the long term. NA on the other hand reduced the rate of hospitalisation. ${ }^{29}$ Cochrane review by Carson-Chahhoud et al compared NA with ICD in the management of PSP in adults $(n=435)$. The review showed better immediate success rate with ICD, reduced length of hospital stay with NA and no difference in early failure rate, 1-year success rate and hospital admission rate, albeit the quality of evidence was reportedly very low to moderate. ${ }^{30}$ A recent systematic review and network meta-analysis of 29 randomised controlled trials $(n=4,262)$ of any treatment in PSP showed that video-assisted thoracoscopic surgery (VATS), ICD and NA had no significant difference regarding recurrence in patients with first episode of PSP. VATS and NA significantly decreased hospitalisation days compared with ICD. ${ }^{31}$
The studies included in these systematic reviews did not look at the subgroup of patients with complete lung collapse separately, as was the case in our study.

A large multicentre Australasian randomised controlled trial is underway, comparing observation with interventional management of large (size $\geq 32 \%$ ) PSP (A randomised controlled trial of conservative versus interventional treatment of primary spontaneous pneumothorax, www.anzctr.org.au/Trial/Registration/ TrialReview.aspx?id=336270). Another multicentre UK trial is comparing standard care of PSP as per BTS guidelines with ambulatory management with the use of an ambulatory device (Randomised ambulatory management of primary pneumothorax (RAMPP) trial, www.isrctn.com/ISRCTN79151659). Recently published data from an interim subgroup analysis of the RAMPP trial indicated that early digital air leak measurements in PSP could potentially predict future medical treatment failure. ${ }^{32}$

\section{Limitations}

The present study has some limitations that should be considered when interpreting the findings.

Like most of the PSP studies, the study size is small. Patient data was collected from case notes and electronic records and the results could have been affected by the omission of unmeasured confounding variables.

This study was a single centre retrospective observational study over a 7-year period, with all the known limitations of retrospective data collection. As the study was not randomised or applied by a strict protocol, treatment allocation could potentially have been a function of circumstances that could include severity and duration of symptoms, patient preference, availability of resources including trained staff at different times of the day and other confounding variables.

There is no clear cut or widely accepted definition of complete lung collapse and we used the criteria laid down by SEPAR. ${ }^{12}$

Another limitation of the study is that the success rate is relatively hard to be defined retrospectively. Well-designed prospective randomised control trials are needed to validate these findings.

\section{Conclusion}

This study is the first of its kind to look at the success rate of NA compared with ICD as the first intervention in PSP with complete 
lung collapse. Our results have shown significantly better success rates with ICD as the first line treatment compared with NA. A considerable number of patients will have persistent air leak despite ICD and will require surgical intervention. PSP with complete lung collapse could be managed as a subgroup in which $N A$ is not attempted. NA remains the first line intervention in PSP patients who are symptomatic and do not have complete lung collapse as it is less invasive, simple to perform and reduces length of hospital stay. A prospective, randomised, multicentre study is required to validate these findings in a larger cohort.

\section{Consent}

No patient information or patient identifiable images are included in this manuscript.

\section{Acknowledgements}

We thank Dr Martin Allen (University Hospitals of North Midlands, Stoke-on-Trent, UK) for his critical appraisal and review of the article.

\section{References}

1 Lesur O, Delorme N, Fromaget JM, Bernadac P, Polu JM. Computed tomography in the etiologic assessment of idiopathic spontaneous pneumothorax. Chest 1990;98:341-7.

2 Melton LJ 3rd, Hepper NG, Offord KP. Incidence of spontaneous pneumothorax in Olmsted County, Minnesota: 1950 to 1974. Am Rev Respir Dis 1979;120:1379-82.

3 Bense L, Eklund G, Wiman LG. Smoking and the increased risk of contracting spontaneous pneumothorax. Chest 1987;92:1009-12.

4 Hallifax RJ, Goldacre R, Landray MJ, Rahman NM, Goldacre MJ. Trends in the incidence and recurrence of inpatient-treated spontaneous pneumothorax, 1968-2016. JAMA 2018;320:1471-80.

5 Gupta D, Hansell A, Nichols T et al. Epidemiology of pneumothorax in England. Thorax 2000;55:666-71.

6 Walker SP, Bibby AC, Halford P et al. Recurrence rates in primary spontaneous pneumothorax: a systematic review and meta-analysis. Eur Respir ] 2018;52:1800864

7 Miller AC, Harvey JE. Guidelines for the management of spontaneous pneumothorax. Standards of Care Committee, British Thoracic Society. BMJ 1993:307:114-16.

8 Henry M, Arnold T, Harvey J, SoCCBTS Pleural Diseases Group. BTS guidelines for the management of spontaneous pneumothorax. Thorax 2003;58(Suppl 2):ii39-52.

9 MacDuff A, Arnold A, Harvey ], Group BTSPDG. Management of spontaneous pneumothorax: British Thoracic Society pleural disease guideline 2010. Thorax 2010;65(Suppl 2):ii18-31.

10 Hooper C, Maskell N. British Thoracic Society national pleural procedures audit 2010. Thorax 2011;66:636-7.

11 Conces D, Tarver R, Gray W, Pearcy E. Treatment of pneumothoraces utilizing small caliber chest tubes. Chest 1988;94:55-7.

12 Rivas de Andres J], Jimenez Lopez MF, Molins Lopez-Rodo L et al. Guidelines for the diagnosis and treatment of spontaneous pneumothorax. Arch Bronconeumol 2008:44:437-48.

13 Baumann MH, Strange C, Heffner JE et al. Group APC Management of spontaneous pneumothorax: an American College of Chest Physicians Delphi consensus statement. Chest 2001;119:590-602.

14 De Leyn P, Lismonde M, Ninane V et al. Guidelines Belgian Society of Pneumology. guidelines on the management of spontaneous pneumothorax. Acta Chir Belg 2005;105:265-7.
15 JPSCLD. Guidelines for pneumothorax treatment: The Japan Society for Pneumothorax and Cystic Lung Diseases. 2nd edn. In: Guidelines Committee. Tokyo: Kanehara; 2009:44-7.

16 Tschopp JM, Bintcliffe O, Astoul P et al. ERS task force statement: diagnosis and treatment of primary spontaneous pneumothorax. Eur Respir ] 2015;46:321-35.

17 Raja OG, Lalor AJ. Simple aspiration of spontaneous pneumothorax. Br J Dis Chest 1981;75:207-8.

18 Harvey J, Prescott RJ. Simple aspiration versus intercostal tube drainage for spontaneous pneumothorax in patients with normal lungs. British Thoracic Society Research Committee. BMJ 1994;309:1338-9.

19 Noppen M, Alexander P, Driesen P, Slabbynck H, Verstraeten A. Manual aspiration versus chest tube drainage in first episodes of primary spontaneous pneumothorax: a multicenter, prospective, randomized pilot study. Am J Respir Crit Care Med 2002;165:1240-4

20 Packham S, Jaiswal P. Spontaneous pneumothorax: use of aspiration and outcomes of management by respiratory and general physicians. Postgrad Med J 2003:79:345-7.

21 Chan SS, Lam PK. Simple aspiration as initial treatment for primary spontaneous pneumothorax: results of 91 consecutive cases. J Emerg Med 2005;28:133-8.

22 Camuset J, Laganier J, Brugiere $\mathrm{O}$ et al. Needle aspiration as firstline management of primary spontaneous pneumothorax. Presse Med 2006;35:765-8.

23 Ayed AK, Chandrasekaran C, Sukumar M. Aspiration versus tube drainage in primary spontaneous pneumothorax: a randomised study. Eur Respir ] 2006;27:477-82.

24 Nishiuma T, Ohnishi $\mathrm{H}$, Katsurada $\mathrm{N}$ et al. Evaluation of simple aspiration therapy in the initial treatment for primary spontaneous pneumothorax. Intern Med 2012;51:1329-33.

25 Thelle A, Gjerdevik M, SueChu M, Hagen OM, Bakke P. Randomised comparison of needle aspiration and chest tube drainage in spontaneous pneumothorax. Eur Respir J 2017:49:1601296.

26 Nikolić MZ, Lok LS, Mattishent K et al. Noninterventional statistical comparison of BTS and CHEST guidelines for size and severity in primary pneumothorax. Eur Respir ] 2015;45:1731-4.

27 Devanand A, Koh MS, Ong TH et al. Simple aspiration versus chest-tube insertion in the management of primary spontaneous pneumothorax: a systematic review. Respir Med 2004;98:579-90.

28 Zehtabchi S, Rios CL. Management of emergency department patients with primary spontaneous pneumothorax: needle aspiration or tube thoracostomy? Ann Emerg Med 2008;51:309.

29 Aguinagalde B, Zabaleta J, Fuentes M et al. Percutaneous aspiration versus tube drainage for spontaneous pneumothorax: systematic review and meta-analysis. Eur ] Cardiothorac Surg 2010;37:1129-35.

30 Carson-Chahhoud KV, Wakai A, van Agteren JE et al. Simple aspiration versus intercostal tube drainage for primary spontaneous pneumothorax in adults. Cochrane Database Syst Rev 2017;9:CD004479.

31 Vuong NL, Elshafay A, Thao LP et al. Efficacy of treatments in primary spontaneous pneumothorax: A systematic review and network meta-analysis of randomized clinical trials. Respir Med 2018;137:152-66

32 Hallifax RJ, Laskawiec-Szkonter M, Rahman NM. Predicting outcomes in primary spontaneous pneumothorax using air leak measurements. Thorax 2018.

Address for correspondence: Dr Muhammad Badar Ganaie, Department of Respiratory Medicine, Royal Stoke University Hospital, University Hospitals of North Midlands, Newcastle Road, Stoke-on-Trent, ST4 6QG, UK.

Email: muhammad.ganaie@uhnm.nhs.uk 